This is the post peer-review accepted manuscript of:

M. Guermandi, S. Benatti, V. J. Kartsch Morinigo and L. Benini, "A Wearable Device for MinimallyInvasive Behind-the-Ear EEG and Evoked Potentials", 2018 IEEE Biomedical Circuits and Systems Conference (BioCAS), Cleveland, OH, 2018, pp. 1-4. doi: 10.1109/BIOCAS.2018.8584814

The published version is available online at: https://doi.org/10.1109/BIOCAS.2018.8584814

(C) 2018 IEEE. Personal use of this material is permitted. Permission from IEEE must be obtained for all other uses, in any current or future media, including reprinting/republishing this material for advertising or promotional purposes, creating new collective works, for resale or redistribution to servers or lists, or reuse of any copyrighted component of this work in other works 


\title{
A Wearable Device for Minimally-Invasive Behind-the-Ear EEG and Evoked Potentials
}

\author{
Marco Guermandi*, Simone Benatti*, Victor Javier Kartsch Morinigo*, Luca Benini* ${ }^{*}$ \\ * Energy Efficient Embedded Systems Lab (EEES), DEI, University of Bologna, Italy \\ ${ }^{\dagger}$ Integrated Systems Laboratory (IIS), ETH Zurich, Switzerland. \\ Corresponding Author Email: marco.guermandi@unibo.it
}

\begin{abstract}
We present an unobtrusive and minimally invasive system for acquiring 8-channels of EEG behind the ear, to support brain activity monitoring in a socially acceptable fashion and outside clinical environments. Electrical performance are in line with those of clinical EEG systems $\left(0.48 \mu V_{R M S}\right.$ integrated noise in the [0-100] $\mathrm{Hz}$ band, $106 \mathrm{~dB}$ CMRR) and setup is extremely fast, requiring only a mild cleaning of the skin before applying the device. The system can acquire typical brain activity rhythms and potentials evoked by sensory stimuli, which are at the basis of BCI systems. Current consumption is $6.2 \mathrm{~mA}$, plus $16 \mathrm{~mA}$ for Bluetooth ${ }^{\circledR}$ data transmission (4.5 hours life on a 100 mAh battery). The overall cost of components is below 50 USD.
\end{abstract}

\section{INTRODUCTION}

Several techniques are available to monitor brain activity (fMRI, PET, DOT, EEG, MEG, ECoG). Among these, electroencephalogram (EEG) recording is considered as the least invasive, the easiest to setup and the one with the lowest cost [1][2][3][4]. This makes EEG the ideal (if not the only) candidate to pursue brain activity monitoring outside clinical environments. In fact, recent times have seen a widening of the fields of EEG application from those typical of the clinical setting to include Brain-Computer Interfaces (BCI), homecare, neurofeedback and gaming [1][4].

Clinical-grade EEG systems aim to provide the most extensive coverage of the head of a test subject, leading to cumbersome systems which are not comfortable and are hardly socially acceptable in a non-clinical environment. Moreover, conductive gel or paste and skin preparation are adopted to improve electrode contact quality. This approach is clearly to be avoided in devices targeting the consumer market [1]. Two types of dry electrodes have been proposed to reduce preparation time [5]. The first type is based on each electrode comprising several pins to penetrate the hair layer. Disadvantages are in terms of limited comfort (as a good amount of pressure is needed to guarantee contact) and difficulties in operation especially for subjects with dense hair. Flat electrodes can be used in non-hairy regions with several consumer EEG systems placing them on the subject's forehead [6]. However, SNR is extremely poor due to the position being close to where the largest artifacts occur (i.e. eye blinking and movement) and far from where most of EEG signal originates. For both approaches, concealing the system is almost impossible.

To overcome these limitations, recent works have proposed to acquire signals in the ear region (so-called ear-EEG), both in-ear [2] or behind-the-ear [7]. Despite the thickness of the temporal bone and the moderate distance from most cortical areas of interest, the technique can be used to acquire EEG signals of use in most consumer applications, namely brain activity rhythms and event related potentials (ERP). While in-ear EEG often requires custom-molded supports, behindthe-ear solutions easily adapt to most subjects and provide maximum unobtrusiveness. They are therefore one of the best candidates for wearable and minimally invasive EEG systems. Disposable wet electrodes for behind-the-ear EEG are commercially available [7], but require preparation and clinical-grade acquisition systems. To our knowledge there is no wearable device integrating reusable dry electrodes and a high-quality acquisition system for behind-the-ear EEG.

We present a prototype of such a system, integrating 8 active dry electrodes for high quality signal pick-up with the shortest setup time, Analog to Digital Conversion (ADC), battery management and Bluetooth ${ }^{\circledR}(\mathrm{BT})$ data transmission to a remote host. It integrates a microcontroller (MCU) for control and embedded computation on a ARM Cortex M4 processor. The device self-detects electrode contact quality, informing a non-trained user of its correct placement, and can receive synchronization signals from the host as needed for ERP acquisition. To minimize area and cost, an external memory is not foreseen as the allocation of SRAM (512 KB) on the MCU is large enough to allow for the few seconds of data buffering needed to process data in the intended applications (i.e. BCI, neurofeedback, gaming...). For testing purposes or continuous monitoring, the prototype can stream raw data for all 8 channels up to $2 \mathrm{ksps}$. Despite size not being minimized in this prototype, the system is small and light enough to be worn comfortably. A lightweight rechargeable 100 mAh LiPo battery allows for 4.5 hours of data acquisition and streaming and more than 4 days stand-by time. The overall cost of components is below 50 USD.

Details of the system design are described in Sec. II, while Sec. III and IV present electrical and functional characterization, showing how the system can acquire rhythms typical of brain activity and event related potentials evoked both by visual and auditory stimuli. In Sec. V we draw some conclusions and outline future work.

\section{SySTEM DESCRIPTION}

The prototype (see Fig.1) can be divided in two parts. A flexible polyimide PCB is used for signal pick-up and 


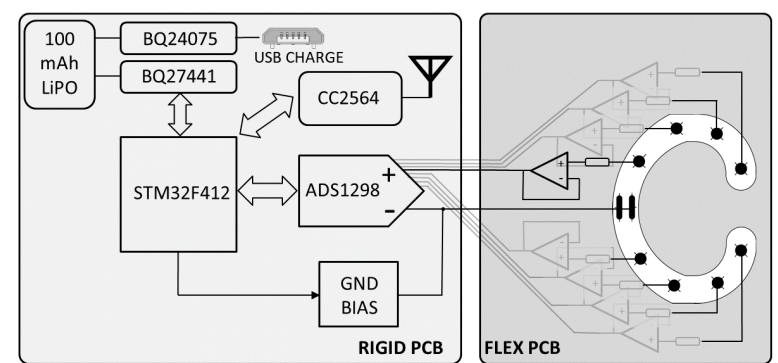

Fig. 1. Main building blocks of the prototype. Buffers are on the flexible PCB containing the electrodes, while the other components are on a rigid PCB.

buffering and presents on one side 8 circular, 5-mm, gold plated electrodes for EEG signals and 2 electrodes for patient ground and reference (see Fig.2, left). To simplify the setup, these electrodes are shorted in the current prototype. Bottom side of the board allocates the active electrode electronics, which make the signal quality resilient to the higher contact impedance of dry electrodes with respect to wet electrodes with skin preparation [3]. The electrodes are covered with commercial reusable adhesive hydrogel patches which also provide support for the device. Double-sided adhesive tape can be added in clear spaces between the electrodes to improve stability. The second part of the system is located in a 3 by $6 \mathrm{~cm}$ rigid PCB and contains the Analog Front End (AFE) for A/D conversion (T.I. ADS1298), BT module and antenna (T.I. CC2564MODA), fuel gauge (T.I. BQ27441) and Li-Ion Battery Charger (T.I. BQ24075). All these peripherals communicate with a STM32F412 MCU which runs the firmware, including the BT stack. A micro-USB port is available for charging the $100 \mathrm{mAh}$ LiPo battery.

\section{A. Signal Acquisition}

As single-ended amplification stages with gain higher than one limit the rejection of common mode noise [3], only signal buffering is performed on the active electrode by an Operational Amplifier (O.A.) connected as a unity-gain buffer. A $68 \mathrm{~K} \Omega$ resistor in series with the amplifier input limits the patient auxiliary current below the applicable limit of 50 $\mu \mathrm{A}$ in single fault condition (i.e. electrode shorted to one of the power supplies). The O.A. is an AD8603 from Analog Devices, showing low voltage noise $(2.3 \mu \mathrm{V}$ peak-to-peak in the 0.1 to $10 \mathrm{~Hz}$ band and $25 \mathrm{nV} / \operatorname{sqrt}(\mathrm{Hz})$ at $1 \mathrm{KHz})$ and a maximum quiescent current of $50 \mu \mathrm{A}$. Input impedance is in excess of $500 \mathrm{M} \Omega$ in the EEG band.

Analog-to-Digital Conversion is performed by an ADS1298 AFE. With respect to ADS1299 which specifically targets EEG signal acquisition, it has reduced power consumption and allows for $3 \mathrm{~V}$ supply operation (removing the need for step-up DC/DC conversion of the battery voltage) without significantly degrading noise performance. The AFE supports sampling frequencies up to $32 \mathrm{ksps}$ with a gain of the input programmable gain amplifier (PGA) from 1 to 12 . The buffers are connected to the non-inverting inputs of the AFE, with inverting inputs tied to the reference electrode. SPI interface is used to communicate with the MCU. In idle state,

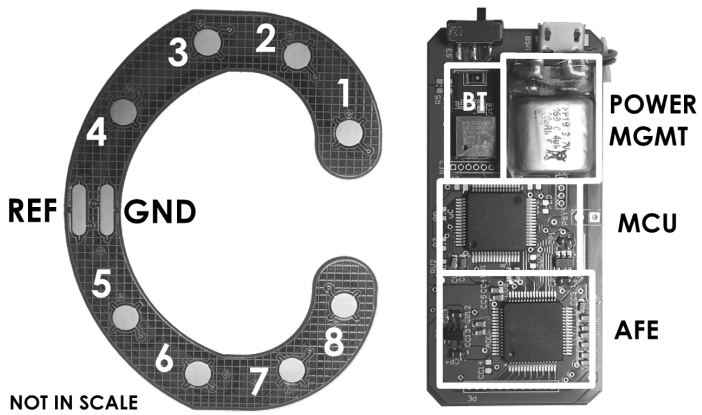

Fig. 2. On the left: Flexible board showing gold electrodes (1 to 8 plus reference and ground). Protection resistors and buffers are placed on the opposite side. On the right: Back-end rigid PCB (3 by $6 \mathrm{~cm}$ size) with main components highlighted. Boards are not in scale.

buffers and AFE are disabled to minimize power consumption.

\section{B. Electrode Contact Check}

The AFE can perform contact impedance monitoring by injecting a small current between two electrodes and measuring the resulting voltage difference. This is a typical approach in systems based on passive electrodes or on active electrodes with custom ICs [3] but is not a viable option in our case, where current sources should be added at the input of each active electrode amplifier, increasing the cost and size of the system. To overcome this limitation, we devised the scheme in Fig. 3, where $\mathrm{Z}_{C 1}$ and $\mathrm{Z}_{C 2}$ represent the contact impedance of the measuring and ground electrodes respectively. $Z_{T}$ is the equivalent impedance of the tissue between the two electrodes and is neglected as significantly smaller than dry-electrode contact impedance.

The system superimposes a $200 \mathrm{mV}$ square wave to the $\mathrm{DC}$ voltage on the ground and reference electrode. As the amplifier is extremely close to the electrode, parasitic capacitance on the electrode will be almost completely due to the input capacitance of the amplifier itself and, as the values of $\mathrm{Z}_{C 1}$, $\mathrm{Z}_{C 2}$ increase, will cause a voltage divider effect which reduces the amplitude of the first harmonic of the square wave. The difference between the full amplitude of the signal on the ground electrode and that detected on the signal electrode provides a measure of the contact quality and is monitored by the AFE. The MCU band-pass filters the digitized signal and computes the average power of the first harmonic of the signal. The higher the value, the higher the contact impedance and, therefore, the worse the contact quality. Contact impedance can be monitored on-line without affecting EEG signal quality given that the frequency of the square wave signal is chosen to be outside the EEG band of interest.

\section{Data Transfer and Synchronization}

Data communication with remote host is guaranteed by T.I. CC2564MODA, a complete BT solution with integrated chip antenna, interfaced to the MCU through a $2 \mathrm{Mb} / \mathrm{sec}$ UART. The remote host can send commands (starting and stopping acquisition, electrode contact checking, programming the AFE) and synchronization signals. Digitized EEG data or 


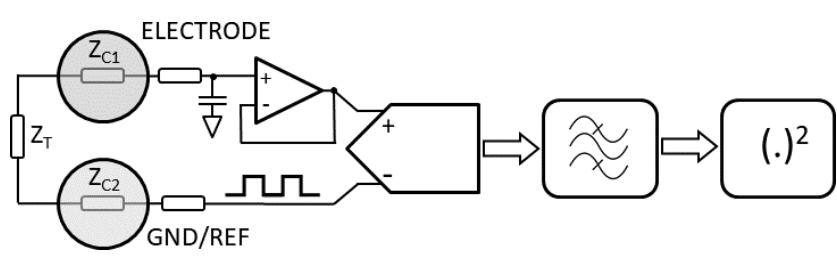

Fig. 3. Scheme of the contact quality check method. A square wave is applied to patient ground; the attenuation of its first harmonic on the electrode is related to voltage divider effect at the input of the amplifier due to finite contact impedances $\mathrm{Z}_{C 1}$ and $\mathrm{Z}_{C 2}$, and provides an estimate of their value.

results of embedded processing and information on system status are sent back to the remote host.

\section{Battery and Battery Management}

Power supply is provided by a lightweight (3 grams), rechargeable, $3.7 \mathrm{~V}, 100 \mathrm{mAh}$ LiPO battery. It can be charged through a USB-micro connector and a T.I. BQ24075 1.5 A LiIon battery charger and power-path management IC. The T.I. BQ27441 fuel gauge is used to regularly check for battery status on a I2C interface. The MCU disables power supply to the analog circuits when the device is in charge to guarantee isolation between subject and mains at any time.

\section{ElectricAl ChARACTERIZATION}

We characterized the system at 500 SPS sampling frequency which guarantees a bandwidth of $131 \mathrm{~Hz}$, already exceeding the needs of our target applications.

Noise is measured by shorting the inputs of the active electrodes and ranges from $1.8 \mu \mathrm{V}_{R M S}$ with PGA gain equal to 1 , down to $0.48 \mu \mathrm{V}_{R M S}$ with PGA gain equal to 12 . Common Mode Rejection Ratio is measured at $92 \mathrm{~dB}$ for differential acquisition between one signal electrode and the reference electrode, increased to $106 \mathrm{~dB}$ when the difference between two channels is computed in the digital domain. Channel isolation is $95 \mathrm{~dB}$. These values are in line with IFCN standards for clinical recording of EEG signals [3].

Table I presents current consumption of the different blocks of the system when in an idle state (BT connected, no streaming, analog circuits off) and signal acquisition. Careful implementation of the firmware (data transfers moved to DMA, clock gating and optimization of clock frequency) allows for a $10 \mathrm{X}$ reduction in the power consumption of digital circuits with respect to that obtained running the MCU at full speed. To simplify testing, we transfer all data to the host device as the prototype is capable of streaming all 8 channels up to $2 \mathrm{ksps}$ ( $\approx 400 \mathrm{kbit} / \mathrm{sec}$ throughput on the BT connection). As we use only $1 \%$ of the total processing capability of the Cortex M4 on the STM32F412, once a specific application is chosen, data processing will be moved to the embedded processor and only its results be streamed to the host.

\section{Acquisition of EEG And Evoked Potentials}

\section{A. Alpha waves}

In the following experiments, the ear patch is applied to a right-handed healthy subject without previous history of neural diseases. In the first test, the subject is asked to keep

\begin{tabular}{|c||c|c|c|c|}
\hline & Analog & Digital & TX/RX & Total \\
\hline \hline Idle & $<50 \mu \mathrm{A}$ & $550 \mu \mathrm{A}$ & $400 \mu \mathrm{A}$ & $1 \mathrm{~mA}$ \\
\hline Acquisition (BT idle) & $3.2 \mathrm{~mA}$ & $2.54 \mathrm{~mA}$ & $400 \mu \mathrm{A}$ & $6.2 \mathrm{~mA}$ \\
\hline Acquisition (BT on) & $3.2 \mathrm{~mA}$ & $2.85 \mathrm{~mA}$ & $16 \mathrm{~mA}$ & $22.1 \mathrm{~mA}$ \\
\hline
\end{tabular}

BREAKDOWN OF CURRENT CONSUMPTION.

his eyes alternatively closed and open for 20 seconds. Alpha rhythm is a meaningful signal for many applications, including drowsiness detection and neurofeedback, and its amplitude increases in eye-closed condition. Fig. 4 shows the signal on electrode 3, where amplitude is maximum; an increase in power by a factor 3 in the alpha band (at approximately 12 $\mathrm{Hz}$ ) is visible when the subject has his eyes closed.
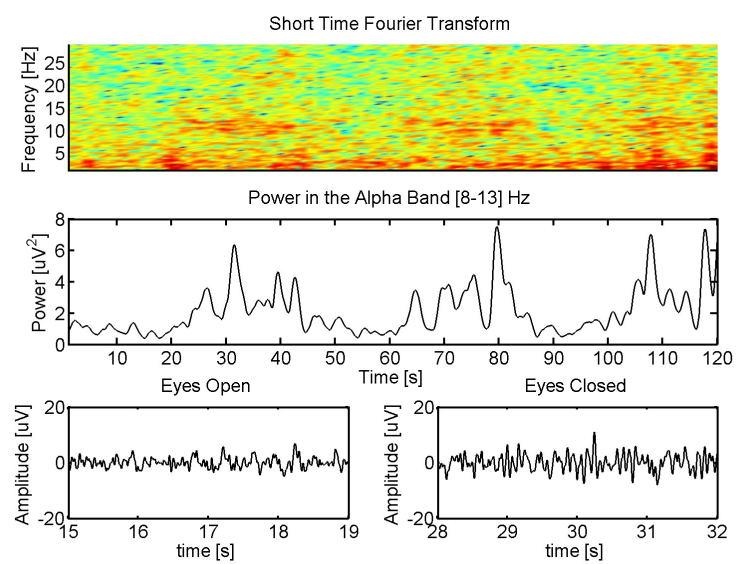

Fig. 4. Alpha rhythm acquired on electrode 3. Signal power in the Alpha band increases by a factor 3 in eye-closed conditions ([20-40], [60 - 80] and $[100-120]$ seconds), as visible in the Short Time Fourier Transform (top), in the average signal power (middle) and in the time-domain (bottom).

\section{B. Visual ERPs and Steady-State VEPs}

We tested the capability of the system to acquire signals needed in the two paradigms most commonly used in BCI spellers. Both experiments rely on the subject sitting in a dimly lighted room, $70 \mathrm{~cm}$ apart from a 24-inch LCD screen. A square $8 \times 8$ black and white checkerboard is presented, flickering at a determined rate which elicits a response in the visual cortex which is synchronized to the stimulus. Fig. 5 shows the ERP obtained in a typical paradigm where we average the response to 100 stimuli presented at time intervals of 1 second (plus a random interval of $\pm 200 \mathrm{~ms}$ to avoid habituation). In spite of the amplitude of the signal being significantly smaller (up to 6 times, depending on component and electrode placement) than ERPs acquired by more invasive systems with electrodes closer to the occipital cortex, standard $\mathrm{P} 1, \mathrm{~N} 1, \mathrm{P} 2, \mathrm{~N} 2$ and P3 components are clearly identifiable as they are at least 5 times larger than residual noise level. The latter component (also known as P300) is of particular interest as its detection is the basis for P300 spellers.

Several SoA BCI systems are based on the presentation of visual stimuli at higher frequencies, whose processing by the visual cortex can be detected through frequency-domain analysis of the acquired EEG signal (steady-state Visual Evoked 


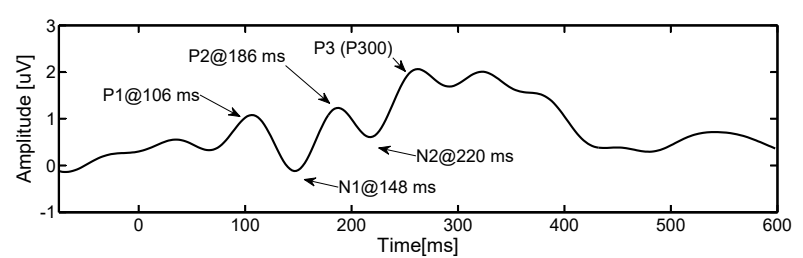

Fig. 5. ERP obtained by averaging 100 epochs of the signal on electrode 3 . All traditional components of ERP are well visible above noise level.

Potentials, SSVEPs). Fig. 6 presents the spectrum of a SSVEP elicited with checkerboards flickering at 10, 12, $15 \mathrm{~Hz}$. On the top row, the spectral line at the stimulus frequency is clearly visible when the Power Spectral Density (PSD) is computed on a 90 -second window, with a SNR of $16 \mathrm{~dB}$, defined as the ratio between the main peak (locked to the stimulus) and the average power in the $10-30 \mathrm{~Hz}$ band. On the bottom row, stimulus frequency is still identifiable for signal windows as small as 5 seconds, although average SNR is reduced to approximately $5.1 \mathrm{~dB}$ which, to be successfully used in BCI systems, calls for methods having better performance than simple frequency tagging, such as Canonical Correlation Analysis (CCA) or mixed phase/frequency approaches.
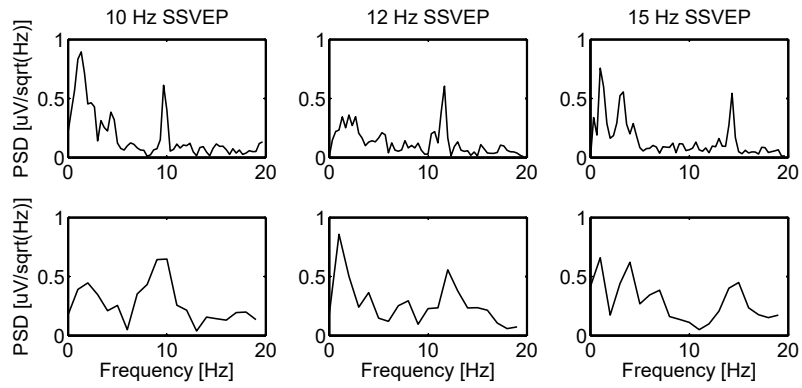

Fig. 6. Spectrum of a SSVEP on electrode 3, elicited by checkerboards flickering at 10,12, $15 \mathrm{~Hz}$. PSD computed on the average of 30 epochs of 3 seconds (top) and 5 epochs of 1 second (bottom).

\section{Auditory Steady-State Response}

Auditory Steady-State Response (ASSR) is very useful, among other applications, to estimate hearing thresholds at different frequencies. We elicited ASSR by providing a test subject with a white noise sound modulated at $40 \mathrm{~Hz}$, while sitting with eyes closed. Fig. 7 shows the PSD for signal windows of 60,20 and 5 seconds on electrode 3 , which is again characterized by maximum amplitude. SNR with respect to a resting state condition is $14.5 \mathrm{~dB}$ in the $60 \mathrm{~s}$ window and is reduced down to $5.5 \mathrm{~dB}$ in the shortest one.

\section{CONCLUSIONS AND FUtURE WORK}

We presented an unobtrusive and minimally invasive system for acquiring 8-channels of EEG behind the ear, supporting efforts to move towards wearable brain activity monitoring systems. Electrical performance is in-line with that of clinical EEG systems $\left(0.48 \mu \mathrm{V}_{R M S}\right.$ noise in the [0-100] $\mathrm{Hz}$ band, 106 $\mathrm{dB}$ CMRR, $95 \mathrm{~dB}$ channel isolation). The setup is fast, as it requires only a mild cleaning of the skin before application, and the device is capable of self-diagnosing electrode contact
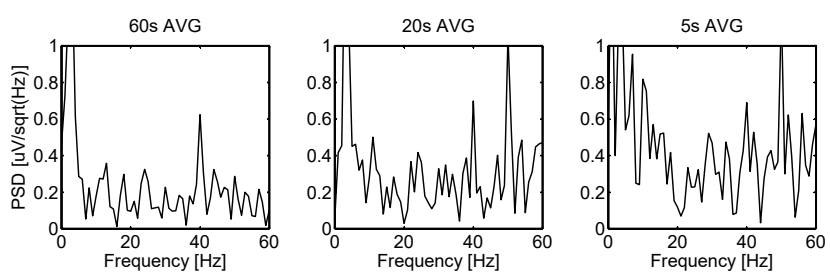

Fig. 7. Spectrum of ASSR elicited with white noise modulated at $40 \mathrm{~Hz}$. Main harmonic is visible in the PSD computed on the average of 60, 20 and 5 epochs of 1 second.

quality. We demonstrated how the system can acquire brain activity rhythms for, e.g., drowsiness detection and neurofeedback, as well as ERPs evoked by sensory stimuli at the basis of most BCIs. To our knowledge, this is the first wearable device worn behind the ear capable of these features.

Current consumption for the acquisition of $8 \mathrm{EEG}$ channels is $6.2 \mathrm{~mA}$, plus $16 \mathrm{~mA}$ for BT data transmission, corresponding to 4.5 hours of operation. As we currently use less than $1 \%$ of the total processing capability of the Cortex M4, once a specific application is chosen data processing can be moved to the embedded processor. As an example, in a SSVEP-based BCI, we can extract features and perform classification on the embedded platform, and transfer only the result. As BT transmission currently dominates power consumption, this can extend battery life up to 16 hours. On the other hand, to compensate for the reduced SNR with respect to more invasive systems, we might need to adopt computational intensive algorithms for pre-processing (e.g. Independent Component Analysis) and feature extraction (e.g. CCA, beamforming) which will account for a major contribution to power consumption. Operational life will then benefit from the use of Ultra-Low-Power architectures for embedded processing. These considerations will guide the development of the system towards a fully autonomous and minimally-invasive solution, which will also include the redesign of the system to minimize size and make it fully conceivable behind the ear of the subject.

\section{ACKNOWLEDGMENT}

This work has been partially supported by the European H2020 FET project OPRECOMP (g.a. 732631).

\section{REFERENCES}

[1] V. Mihajlović et al., "Wearable, wireless EEG solutions in daily life applications: what are we missing?" IEEE journal of biomedical and health informatics, vol. 19, no. 1, pp. 6-21, 2015.

[2] G. Li et al., "Emotion recognition based on low-cost in-ear EEG," in Biomedical Circuits and Systems Conference (BioCAS), 2017 IEEE. IEEE, 2017, pp. 1-4.

[3] M. Guermandi, R. Cardu, E. F. Scarselli, and R. Guerrieri, "Active electrode IC for EEG and electrical impedance tomography with continuous monitoring of contact impedance," IEEE transactions on biomedical circuits and systems, vol. 9, no. 1, pp. 21-33, 2015.

[4] J. Minguillon et al., "Trends in EEG-BCI for daily-life: Requirements for artifact removal," Biomedical Signal Processing and Control, vol. 31, pp. 407-418, 2017.

[5] M. A. Lopez-Gordo et al., "Dry EEG electrodes," Sensors, vol. 14, no. 7, pp. 12 847-12 870, 2014.

[6] R. Maskeliunas et al., "Consumer-grade EEG devices: are they usable for control tasks?" PeerJ, vol. 4, p. e1746, 2016.

[7] M. G. Bleichner and S. Debener, "Concealed, unobtrusive ear-centered EEG acquisition: cEEGrids for transparent EEG," Frontiers in human neuroscience, vol. 11, p. 163, 2017. 